\title{
Monitoring application of distributed optical fiber measurement technology in petrochemical industry
}

\author{
Jianfeng $\mathrm{Gao}^{1,}$ a and Yawen Cai ${ }^{2}$ \\ ${ }^{1}$ School of Petrochemical and Energetic Engineering, Zhejiang Ocean University \\ ${ }^{2}$ Oil and Gas Storage and Transportation Technology Engineering Laboratory of Zhejiang Province
}

\begin{abstract}
With the continuous development of optical fiber measurement technology, it has been more and more used in petrochemical industry. This paper introduces the using two application examples, including distributed optical fiber measurement technology for storage tank temperature and pipeline strain monitoring. Distributed optical fiber temperature measurement DTS application in the crude oil tank fire online monitoring and brillouin BOTDA distributed optical fiber sensor applied in on-line monitoring of submarine pipeline are respectively introduced in detail.Technical advantages of two kinds of optical fiber measurement technology, technical parameters and design scheme are introduced respectively, and combining the specific example of DTS and BOTDA are also analyzed in practical application.
\end{abstract}

Keywords: distributed optical fiber temperature measurement; brillouin distributed optical fiber sensor; on-line monitoring.

\section{Introduction}

Petrochemical industry is one of the important pillar of the national economy. Due to the inherent flammable and explosive, poisonous and harmful characteristics of dangerous chemicals, the safety problem has become particularly important. With the rapid development of petrochemical industry in China, the safety of petrochemical production situation is increasingly serious, especially on the aspects of safety, health and environment issues, which increasingly bring prominent and potential threats. And with the increasingly reserves of crude oil, product oil ,the number of large domestic oil depots, oil tank areas also presents a increasing tendency. The petrochemical industry departments and enterprises at all levels always attach great importance to the security of oil storage and transportation .

\section{The application of distributed optical fiber temperature measurement DTS online monitoring in the crude oil tank}

According to specification for design of petroleum repertory (GB50737-2011), the fire automatic measuring device should be set on the tank, and the area of the fire detector should be divided in

\footnotetext{
a Corresponding author: gaojf309@126.com
} 
accordance with the requirements of fire extinguishing system linkage control. When using optical fiber type heat detector, optical fiber thermal detector should be set on the top of the oil tank floating disc secondary sealing ring. Optical fiber type heat detector has two kinds: 1 . The fiber Bragg grating FBG; 2. The distributed optical fiber temperature measuring DTS.

\subsection{Fiber Bragg grating FBG}

This technique is an earlier study, which many institutions have done a research. At present, the fiber Bragg grating detector is being used in the tank fire monitoring of a lot of domestic crude oil storage. However, it deficiencies in: (1) Poor reliability of sensor. Temperature sensor is concatenated, and melting point is affected by heat bilges cold shrink and it also exists poor reliability when flooded in water , which easy to cause broken fiber sensor failure, lose some or even all of the monitoring area; (2) Measurement is a blind area. Dot distribution measurement only can measures the installation position and the nearby of sensor. Once the temperature sensor is not installed in the position nearby, the temperature cannot be detected effectively. (3)Late operations from volume. Restricted by sensor design and packaging process, the temperature sensor sometimes drifts. Signal demodulation based on Angle tuning meter exists poor long-term reliability, and need regular calibration again, running maintenance workload. Figure 1 is schematic diagram for the fiber Bragg grating FBG application.

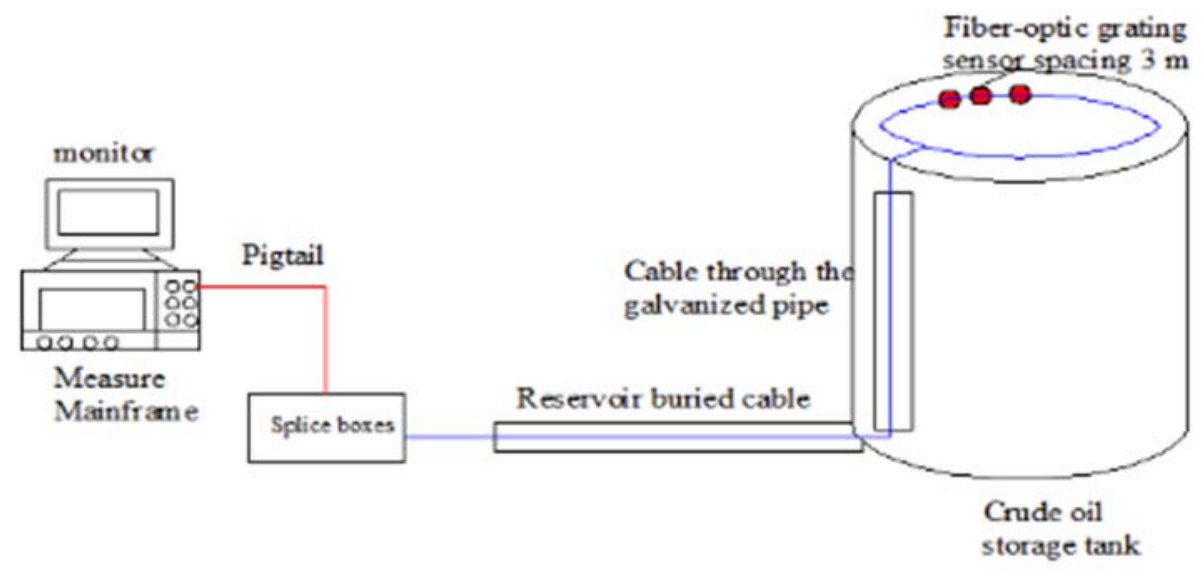

Figure 1. Schematic diagram for the fiber Bragg grating FBG application.

\subsection{Distributed optical fiber temperature measuring DTS}

The basic working principle of distributed optical fiber temperature measurement DTS is: by the optical fiber temperature measurement host according to certain transmitted pulse repetition frequency (PRF) incident light to the sensing optical fiber, produced by the laser pulse and the optical fiber molecular interactions (Raman) Raman scattering effect, after a Stokes light than the light wavelength long (Stokes) and a shorter than light wavelength Anti - Stokes light (Anti - Stokes) along the sensing fiber to launch after back to the optical fiber temperature measurement host, Anti Stokes light signal strength is related to temperature, Stokes light signal has nothing to do with temperature. So, from the sensing optical fiber inside any Anti - Stokes signal and the stokes light signal intensity ratio, the temperature of the access to that point. And space distance of heat, can take advantage of the optical time-domain reflectometer (OTDR) technology by thermal light waves in optical fiber transmission speed and accurate to the scattered light return time after calculation.

\subsubsection{Technical advantages}


(1) Do not need additional sensors; optical fiber is the sensor, realizing the "pass", "feeling".

(2) Fiber has a longer life, smaller size, lighter weight, and the late maintenance workload is small.

(3) Suitable for monitoring under strong electromagnetic fields such as high voltage, resistant to electromagnetic interference.

(4) Higher sensitivity, wider dynamic range, and suitable for remote transmission and monitoring.

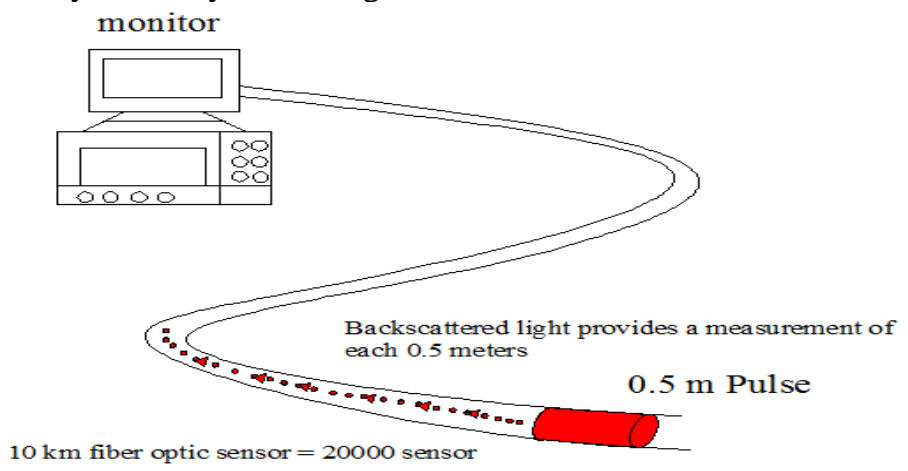

Figure 2. The principle for distributed fiber optic DTS measuring.

\subsubsection{The main technical parameters of DTS}

The parameters of DTS technology include measuring distance, measurement channels, measuring time, temperature measurement precision, temperature resolution and space resolution, sampling interval and positioning accuracy. Technical indicators constrain each other , and measurement time, the temperature resolution, space resolution are the key indicators for monitoring application of oil depots .

(1)Space resolution, namely the minimum heat length, the main index is:

1.Step temperature method (Figure 3) : 10\% - 90\% of the difference in temperature $\mathrm{T}$ is the optical fiber length.

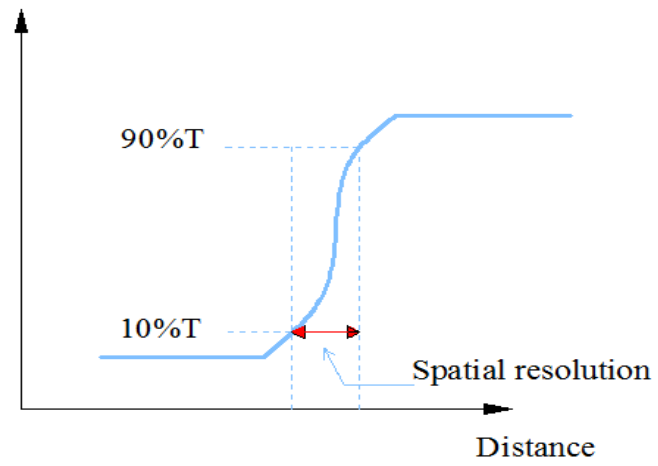

Figure 3. Step temperature method

2.The method of optical fiber around (Figure 4) : put together a few different lengths of optical fiber ring in hot water tank, and the length just equals the maximum temperature of the ring optical fiber length, which is the spatial resolution device. 


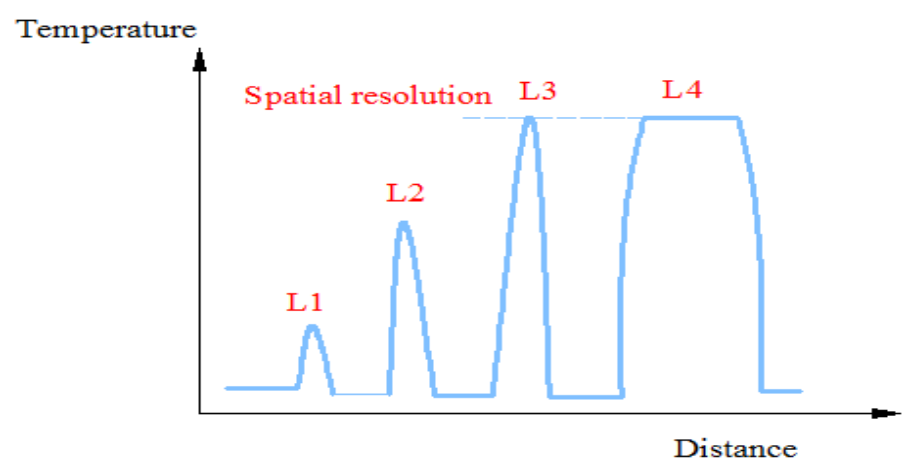

Figure 4. The method of optical fiber around

\subsubsection{DTS design ideas}

(1) Stable: using mature technology products;

(2) Reliable: redundancy backup design (make sure that all the host, raster fault do not affect the real-time monitoring of the whole system);

(3) Rapid: fast heat conduction type of sensing grating;

(4) Simple: visual and intuitive software interface.

\subsubsection{DTS design goals}

(1) The temperature of the real-time online monitoring;

(2) Provide early detection of fire danger;

(3) Clearly introduce the dangerous place;

(4)Put the alarm signals to the control room, and inform the personnel on duty to deal with emergency or fire alarm control system, linkage control.

\subsubsection{System design scheme}

One real-time hosts monitors four tanks, and backup monitoring four tanks; Each tank independent sets two laps sensor cable (double loop design); Both ends of the sensing optical cable come back to measuring unit (circuit design); When a fiber is broken or a host fails, the software can automatically control survey host.

\section{Brillouin type BOTDA distributed optical fiber sensor applied in on- line monitoring of submarine pipeline}

Submarine pipeline connecting the upstream and downstream of offshore oilfield development, is an indispensable key offshore oil and gas field development and production project. The characteristics of submarine pipeline are: 1 . The large investment, high risk; In the event of accident, easy to cause significant loss and environmental disaster. 2. The pipe at the bottom of the sea, the sea bottom in the most need to be buried in silt certain depth, which brings difficulties of inspection and maintenance . 3 . The outside tube electrochemical corrosion, foreign object impact, factors such as wave and seabed movement will also increase the probability of pipeline failure. 4. In tidal range or wave fracture zone pipeline (especially the riser) is easy to be affected by the wind and waves, tide, such as ice, sometimes may be caught in the sea and the ship collision or anchor damage. 


\subsection{BOTDA distributed optical fiber sensor (Brillouin type)}

It is a new kind of real-time online monitoring technology, and the pipe lays along the detection cable in parallel, which can realize along the pipeline vibration, the leak, hot spot and other abnormalities in real-time monitoring. The new technology has the function of longer measuring distance, continuous distributed measurement, more accurately positioning, and the advantages of simpler installation. There is also no any damage or effect to the normal production for buried pipeline, happened to have stable and new leaks to be identified, particularly suitable for pipeline on-line monitoring application.

The sensors, laying with the communication optical cable that pipeline ditched, can get along information of oil and gas pipelines status in real time, such as temperature, strain, vibration. Through analysis of $\mathrm{t}$ he historical data, the extraction of feature signal and the intelligent recognition, implementation of pipeline leakage, pipeline span, third-party damage of pipeline, pipe deformation and geological disaster can early be warned in advance, reaching the goal of prior to predict the time of the incident, places, events, trends, etc., and accurate positioning.

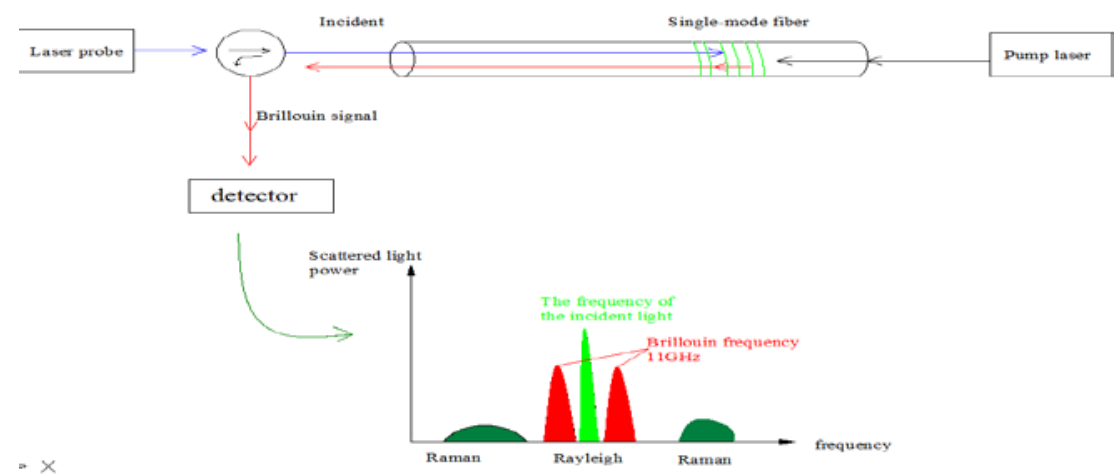

Figure 5. System structure for BOTDA

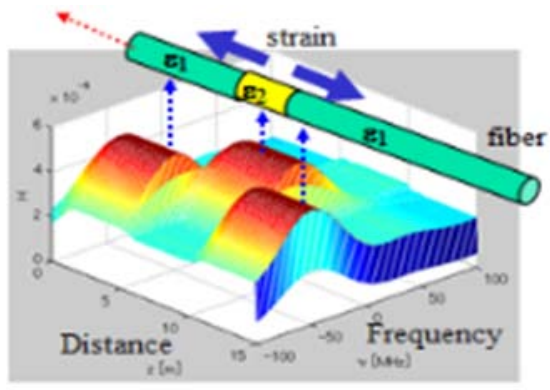

Figure 6. Scattering spectrum for BOTDA

Figure 5 is system structure for BOTDA, Figure 6 is scattering spectrum for BOTDA . It can realize the measurement of temperature along the optical distribution and strain measurement .The current fields of application has been widely included in the undersea optical cable, long-distance oil and gas pipelines, structural health monitoring in the. Looking from the frequency spectrum, brillouin frequency shift is proportional to the strain and temperature change. When optical fiber is changed by different pressure and temperature, fields of brillouin scattering spectrum will drift.

\subsection{Application cases abroad of BOTDA}

(1)Dalia oilfield uses Intergriti Platinum fully integrated optical fiber monitoring system, helping the pipeline operators monitor the temperature, strain and vibration of pipeline. On the pipeline in the process of optical fiber, Dalia oilfield technical team adopted blowing method of fiber placement. Starting from the drilling platform in the submarine pipeline surface preset a along the tube bundle 
spiral stainless steel tubes, and the ends of microtubules back into platform or on the ground, forming a long loop, after being set bundle installation, introducing fiber from one end of the spiral tubes, make it down to the seabed and return to the tanker.

(2) Case of Omnisens company.

Project is located in the UK Lerwick Islay oilfield, using BOTDA to realize heating pipe temperature monitoring. Distributed temperature data through a pipeline, the exploration of $6 \mathrm{~km}$ submarine pipeline exists five risk points.

\section{Conclusions}

Distributed optical fiber temperature measurement can realize real-time online temperature monitoring, can provide early detection of fire danger, can clearly give instructions, where fire risk and alarm signals to the control room, and also can deal with emergency for the personnel on duty or control fire alarm system and linkage. Distributed optical fiber temperature measurement system belongs to the intrinsically safe types, suitable for working in flammable, explosive and dangerous environment, especially suitable for measuring oil tank temperature and the fire alarm.

Distributed optical fiber sensing technology is a new kind of real-time online monitoring technology, the parallel detection cable along the pipe laying, which can realize along the pipeline vibration, the leak, hot spot and other abnormalities in real-time monitoring. It also has the function of measuring distance, continuous distributed measurement, accurating position, simple installation, safe and reliable, strong expansibility, etc, for buried pipeline, it does not produce any damage or affect the normal production, happened to have stable and new leaks that can be identified, particularly suitable for pipeline on-line monitoring application.

\section{Acknowledgements}

This work was supported by the public benefit foundation of Science and Technology Bureau of Zhejiang Province of China (No. 2015C31014).

This work was also supported by the Science Research start-up foundation of Zhejiang Ocean University.

\section{References}

1. Deming Liu , Qizhen Sun . Distributed optical fiber sensing technology and its application [J]. Laser and optoelectronics progress, 11, (2009).

2. Junping Geng, Jiadong $\mathrm{Xu}$, etc. Based on the progress of the brillouin scattering distributed optical fiber sensor [J]. Journal of testing technology,16, 2(2002).

3. Lijun Chen , Haibo Li , etc. The distributed optical fiber temperature measurement technology research status and development trend of [J]. Journal of chemical industry automation and instrumentation, 37, 12(2010).

4. Zhigang $\mathrm{Qu}$. Distributed optical fiber long distance oil and gas pipeline leak detection and early warning technology research[D], (2007).

5. Qizhen Sun . Distributed optical fiber sensing and information processing technology research and application[D], (2008). 